\title{
A Study on the Spread of Chaozhou Opera in Thailand under the Strategy of Maritime Silk Road
}

\author{
Lei Miao \\ GDUFS School of Interpreting \& Translation Studies, Guangdong University of Foreign Studies, Guangzhou, China \\ Qiuping Wang \\ Faculty of Chinese Language and Culture, Guangdong University of Foreign Studies, Guangzhou, China
}

\begin{abstract}
Chaozhou Opera, selected in the first batch of national intangible cultural heritage protection list, is an ancient Chinese opera. It has been spread overseas with Chaozhou people and has become one of the most influential local opera in China, also homesickness sustenance for many overseas Chinese people. In 2013, the General Secretary Xi Jinping proposed the strategy on the construction of the "21st Century Maritime Silk Road", which has clearly pointed out to strengthen the cultural communication and cooperation in the "Maritime Silk Road" regions. Thailand is an important country on the Silk Road. Therefore, this article takes the development of Chaozhou Opera in Thailand as the research object, analyzes the present situation of Chaozhou Opera in Thailand, points out its bottleneck and puts forward the development strategy.
\end{abstract}

Index Terms-Maritime Silk Road, Chaozhou Opera, Thailand, spread

\section{INTRODUCTION}

Chaozhou Opera is one of China's top ten operas and was selected in the first batch of national intangible cultural heritage protection list. Chaozhou Opera, a kind of ancient local opera singing in Chaozhou accent, is popular in the area of guangdong, southern fujian, Taiwan, Hong Kong and southeast Asia. Due to the rise of the Maritime Silk Road, the Chaozhou Opera has become one of the most influential local operas in China. But it only as a "foreign culture" in southeast Asia, its existence form by local social, political, economic, and mainstream culture rules guide and restriction of various factors, which assumes the trend curve. In order to actively respond to the strategy of "the 21st Century Maritime Silk Road" and inherit Chinese culture better on the Silk Road economic belt, this article takes the development of Chaozhou Opera in Thailand as the research object and explores the sustainable development of the spread of Chaozhou Opera in Thailand.

\section{THE ORIGIN OF THE CHAOZHOU OPERA ENTERING INTO THAILAND}

As an important emotion of national sentiment, Chaozhou Opera has a special significance and value for overseas Chinese people. The spread of Chaozhou Opera focus on southeast Asia, especially in Thailand. Therefore, Thailand is also known as the second home of Chaozhou Opera. Chaozhou Opera is able to enter Thailand and spread widely with unique its advantage and this advantage can be reflected in the following three aspects:

First of all, China and Thailand are friendly neighbors since ancient times. According to historical records, the earliest communication between China and Thailand can be traced back to the Western Han Dynasty when China sent messengers to India, passing through the south of Thailand's Siam Bay. This was the earliest history of communication between China and Thailand which could date back more than two thousand years ago. During the Ming and Qing Dynasties, the exchanges between China and Thailand reached a peak. According to "The History in Ming Dynasty", "The Records of the East and the West" and other ancient records, China and Thailand had exchanged emissaries more than 128 times in the Ming Dynasty, in which Thailand sent to the Chinese emissaries102 times. In the Qing Dynasty, from Shunzhi to Thailand Ayu Wang Tuo period, Thailand sent emissaries to Chinese a total of 44 times. Accompanied by the official communication, the friendly exchanges among the people in the two countries had also reached a climax. In the late Qing Dynasty, China's national power began to decline and the war was successive. A large number of Chaoshan people located in the southeast coastal areas began to make a living overseas and Thailand was the destination for most of them. The descendants of these Chaoshan people gradually took root in Thailand and became a new generation of Thai people.

Secondly, Chaoshan has the advantages in natural geography. In the process of cultural communication, the geographical environment is also an important factor. Chaozhou Opera originated in the area of Chaoshan, Guangdong which is located in the southeast corner of the mainland China and at the junction of Guangdong and Fujian, only one thousand kilometers away from Thailand and sharing the same ocean with many Southeast Asian countries. Superior 
geographical conditions provide the possibility for Chaoshanese ancestors to make a living overseas. At the same time, Thailand and the Chaoshan area are close to the climate, so many Chaoshan people choose to migrate in Thailand.

Finally, Chaoshan has the advantages in cultural background. The unique Chaoshan culture, with the characteristics of diversity and inclusiveness, is created by history, economy, geographical environment and many other factors. Not only Chaoshan words is influenced by Minnan dialect, Chu dialect, Malay and many other systems, but Chaoshan is also a multi-ethnic area, including the She ethnic group, Fu Lao people, Han nationality and so on. At the same time, Chaoshan area also has obvious marine culture characteristics. Chaoshan people pay attention to commercial civilization and are more willing to be engaged in business, which makes them have a close communication with people overseas. In addition, the hedonic Chaoshan culture also attracts many audiences. These cultural origins are also important reasons for the Chaozhou Opera to accompany Chaoshan people into Thailand.

\section{The Present Situation of ChaOzhou Opera In Thailand}

Hundreds of years ago Chaozhou Opera was spread to Thailand with the footprints of Chaoshan people, so it is a cultural tie connecting Chaoshan people both at home and abroad. It has a considerable influence on the Chinese ethnic groups in Thailand. The present situation of Chaozhou Opera in Thailand has the following characteristics:

\section{A. An Important Link in the Transmission of National Spirit}

As an important node of the Maritime Silk Road, Chaoshan area plays an important role in the connection from China to Africa, Southeast Asia and South Asia. Chaoshan people brought overseas not only China's property, but also Chaoshan culture, and Chaozhou Opera was one of them. In the moment, Chaozhou Opera, the national spiritual ties, plays an increasingly prominent role. In recent years, the Sino-Thai interaction has been significantly strengthened both in scale and in impact with Chaozhou Opera as the medium. From April 26, 2016 to May 6, the Chaozhou Opera troupe of Chaozhou City held a concert in Bangkok, Thailand. They brought there some classical repertoire such as "Lotus Lantern" and "Mo Chou Nv", as well as some recent created and award-winning new works, such as "The love Story of Cao Ying" and "Han Yu's Governance on Tide'. The national level actor Zheng Shunying and the well-known Li Yulan and XuJiana, as well as a number of bright younger generation had acted in the troupe. In addition, Chaozhou municipal government is also actively promoting the inheritance and development of Chaoshan culture in Thailand. In May 2016, the Chaozhou municipal government carried out the "Chaozhou Festival" in Thailand, and organized the opera performance for a few days. Also some Chinese representatives in Thailand came to China to exchange the culture. In October 2016, Chairman Huang Guoguang of the Chaozhou Guildhall in Thailand led the delegation to Jieyang to hold a communication. Jieyang City Mayor received them and expressed the hope to promote economic prosperity and development between the two counties and achieve mutual benefit and win-win situation under the strategy of "One Belt and One Road Initiative". In this exchange, the delegation of Thailand not only appreciated Chaozhou Opera performances, but also donated 100,000 to the Jiedong tide company donated 100 thousand yuan to Chaozhou Opera troupe of Jiedong City. With the growth of overseas Chinese and the adjustment of national strategy, Chaozhou Opera, as the carrier of national spirit, plays a more obvious part in the process of communication in Thailand.

\section{B. The Lack of Successors of Chaozhou Opera}

The lack of excellent actors of Chaozhou Opera is a prominent problem for the spread of Chaozhou Opera in Thailand. There are two main reasons for the emergence of actor problems. On the one hand, it is influenced by the actor's source. With the departure of the previous generation of Chaozhou Opera, the problem of the source of the new generation is becoming more and more obvious. The loss of the Chinese actor of Chaozhou Opera is serious. The actors of Chaozhou Opera have a low income, and Chinese families in Thailand usually have a better economic condition, while young people are not willing to learn acting, so there is a lack of young Chinese actors who can speak Chaoshan dialect. In order to alleviate this problem, many troupes have to hire native persons of Thailand to perform, but these actors inevitably have language barriers. Although they are also hard-working to memorize the script through Thai phonetic way,the effect of opera performance can inevitably be discounted. On the other hand, there exists the actor's training problem. The development of Chaozhou Opera in Thailand depends on the excellent actors, but the training of the actors also plagued many Chaozhou Opera troupes in Thailand. Lack of professional training on the actors of formal Chaozhou OperaSchool, the role of Chaozhou Opera cannot be played fully. As many Chaozhou Opera actors in Thailand said, now they studied Chaozhou Opera mainly by themselves, unlike the previous time when the teacher would tell them how to do. In addition, in the past Chaozhou Opera attracted the audience by its superb singing and performing skills, but now troupes mainly depends on exaggerated makeup and colorful clothing to attract viewers. As a result, some actors no longer pay attention to the study of performance skills.

\section{The Weakness of the New Generation of Audience}

With the development of the times, the number of young Chinese in Thailand understanding Chaoshan dialect is becoming less and less. The young Chinese in Thailand is becoming more and more unfamiliar to the cultural traditions of Chaozhou Opera. The problems among the audience in Thailand are another challenge in the process of the spread of Chaozhou Opera. Chaozhou Opera was ever popular in Thailand. The old busy theater is now sluggish. Compared to 
Chaozhou Opera, the younger generation of overseas Chinese prefers the tradition of Chaoshan Kung Fu tea, which is easier to accept, and more easily inherited. In an influential Chaozhou Opera troupe in Thailand named "Qingnang Yulouchun, its boss said that now there were less and less people who liked Chaozhou Opera and the troupe was absolutely no profit. Their insistence on the troupe is more out of love for their homes and Chaozhou Opera, while the troupes' maintenance will need it to support to other businesses. The loss of Chinese viewers and the language barrier of the Thai audience have become another bottleneck in the development of Chaozhou Opera in Thailand.

\section{The CAuse of the Development of the ChaOzhou Opera}

The development of chaozhou opera in Thailand has been both effective and challenging. This is closely related to the external environmental factors and the internal causes of the drama itself, and the main causes are the following three aspects.

\section{A. Thailand's Moderate National Politics Has Boosted the Chinese Identity with Thailand}

Thailand has adopted a policy of identification with ethnic Chinese on the national policy. They encourage Chinese people to marry thais and create a harmonious and free business atmosphere. Chinese people in Thailand are treated equally with the Thai people. The Thai government, under the open policy of Chinese in Thailand's political and social life shall enjoy the equal rights, therefore, the Chinese in Thailand life more calm and confident, Thailand's tolerance and acceptance, makes the Chinese population will be easier to "home" as a "new home". This kind of "assimilation" plays down the support of the Chaozhou Opera in the current transmission of Thailand from national identity, especially among the young people.

\section{B. The Current Education Is Lacking in Chinese Education}

Although there are not obvious faults in Chinese education in Thailand, The current education is lacking in Chinese education. In the 1940s, the government had imposed strict restrictions on Chinese schools, and nearly a hundred schools in Thailand were closed. By 1951, only two hundred Thailand throughout the rest of the Chinese schools, however, the Thai government to these Chinese schools also conducted a variety of restrictions exist in name only, including teaching subjects and teaching time and so on are defined. This series of blows has caused the decline of the whole Thai language education, especially in the intergenerational transmission of Chinese language. Although some qualified Chinese families take the form of Chinese family class, it has little effect, and only some Chinese can afford such expenses. Bound for the spread of Chinese language, therefore, directly affect the spread of Chaozhou Opera.

\section{The Inevitable Shackles of the Chaozhou Opera in the Course of Development}

As a local opera, the audience of Chaozhou Opera not only of Chinese people but also of indigenous people. However, in the process of the spread of the drama, the language and the geographical scope of the audience have certain requirements for the audience. Traditional Chaozhou Opera, the content allusions are also related, these factors have become the inevitable shackles in the overseas communication process. How to solve these problems is a problem that the tide drama must face and solve abroad.

\section{The Development Strategy of Chaozhou Opera in Thailand under the Strategy of Maritime Silk ROAD}

Chaozhou Opera bears the important task of cultural heritage in Thailand, but the current spread situation in Thailand is not optimistic. With the increase in the number of new Chaoshan immigrants, the role of the future Chaozhou Opera, as a national spiritual tie, will be even more important in Thailand. How to promote the development of Chaozhou Opera in Thailand under the strategy of the 21st Century Maritime Silk Road and make this excellent national culture get better inheritance becomes urgent problems to be solved.

A. Combining the Strategies of "Going out" and "Come in"

The sustainable development of Chaozhou Opera in Thailand can not be separated from the support of the birthplace of drama. This "Going out" and "Coming in" model should continue to be improved both in quality and quantity in the future. Chaozhou Opera origins from Chaoshan area where the art of Chaozhou Opera is developed and all cities have their own art troupes and excellent actors of Chaozhou Opera. The artists of Chaozhou Opera both in China and Thailand should deepen exchanges, learn from each other and get more artistic wisdom. In China, Chaozhou Opera has gotten good development and protection. In 2006, Chaozhou Opera was selected in the first batch of national intangible cultural heritage protection list. From 2011, Shantou City has held five consecutive "International Festival of Chaozhou Opera". The number of art troupes coming to China is increasing each country. Chaozhou Opera troupes in Thailand should take the active action to invite Chaozhou Opera troupes in China to have a visit and exchange in Thailand, thus bring high-quality performance to Thailand, enhancing the influence of Chaozhou Opera, and actively learning from the valuable experience. At the same time, actors of Chaozhou Opera in Thailand should go to China and other regions to study how to inherit the art of Chaozhou Opera better and how to develop the value of Chaozhou Opera better. In China, the development and protection of Chaozhou Opera is not just at the artistic level. In order to make Chaozhou Opera 
create more value, Chaoshan area has done a lot of attempts. The theme park of overseas Chinese culture has been completed in Shantou Special Economic Zone, and it will gather Chaozhou Opera and other folk culture together to form a industrial chain model of a set of tourism, real estate and business exhibition so that under the market economy environment, Chaozhou Opera can still create high commercial value. In addition, the Shantou city government also integrated Chaozhou Opera and other cultural resources, and actively participated in the display of various platforms to get more attention for Chanshan opera and attract more investment. In 2015, Shantou city Government participated in the "China International Fair" and tried to build a Shantou Museum on the theme of "Reviving the Maritime Silk Road and Developing Shantou Special Economic Zone". The museum attracted many domestic and foreign buyers and visitors and got more attention for the development of Chaozhou Opera. These Chinese experiences are very valuable for the artists of Chaozhou Opera in Thailand to have a visit and study by themselves. At the same time, Chaozhou Opera was also spread to Singapore, Indonesia and other places and the spread of Chaozhou Opera in these exotic spreads is also a valuable experience for the development and study of Chaozhou Opera troupes in Thailand.

\section{B. The Innovation of the Spread Form of Chaozhou Opera in Thailand}

With the development of the times, the vitality of the drama is easily to decline, nut innovation can provide an inexhaustible motive force for the drama. For any kind of excellent culture, if it wants to be deeply rooted in the land of another nation, it must first adapt to the local national psychology, national character and habit. In addition it must also find an expression form suitable for the nation, because just in this way will it not dry up when leaves its own birthplace. These are the directions where Chinese Chaozhou Opera needs to innovate in the process of the spread in Thailand in the future.

A lot of far-sighted artists of Chaozhou Opera in Thailand are trying to make Chaozhou Opera more adaptable to this piece of soil through the way of innovation, and ZhuangMeilong, the artist of Chaozhou Opera in Thailand, is one among them. Zhuang Meilong believes that in order to continue to get promoted in Thailand, this excellent Chinese opera-Chaozhou Opera must try to attract not only the Chaoshan Chinese overseas but also the local audience in Thailand. For the local audience in Thailand, the language is the biggest problem. In order to overcome this difficulty, some troupes had used "simultaneous interpretation" to resolve it, but this approach made the effect of Chaozhou Opera greatly reduced in the artistic atmosphere. Zhuang Meilong conducted on another attempt in which based on Chinese Chaozhou Opera,he tried to absorb the essence of other Chinese opera and integrate the local culture and art in Thailand. He used Thai language to sing Chaozhou Opera and made a bold innovation. After a constant attempt and accumulation, the Chaozhou Opera in Thai language was finally born. This attempt has opened up a new space for the spread of Chaozhou Opera in Thailand and gotten the recognition from Thai Princess Sirindhorn.

The artists have done more than that for the innovation of the spread of Chaozhou Opera in Thailand. They adapted Chaozhou Opera into TV series and made Chaozhou Opera get more attention of the ordinary people in Thailand. In recent years, more and more TV series on the theme of Chaozhou Opera constantly appear on the screen, and these TV series have reached a certain height both in quality and lineup, among which "The Last Peony Flower" is one representative. "The Last Peony Flower" is based on Chaozhou Opera troupe and arranged according to the aesthetic taste of people in Thailand. It also invited Thai popular stars Aum Atichart and Aff Taksaorn. This opera won the Best Drama Award of the year, and the actor Aum Atichart won the title of best actor of the year.

To some extent these innovations has injected new vitality for the development of Chaozhou Opera in Thailand. However, if Chaozhou Opera wants to get sustainable development in Thailand, only by these innovations is not enough. Chaozhou Opera still need to constantly innovate the form, content and the way of acting in the future development to adapt to changing circumstances. At present, the spread of Chaozhou Opera in Thailand faces both challenges and opportunities. In this situation, Chaozhou Opera must actively adjust itself, otherwise it will be eliminated by the age.

\section{Seizing the Opportunity and Seeking Support}

In 2013 General Secretary Xi Jinping proposed the strategy of the 21st Century Maritime Silk Road which has pointed out clearly the need to strengthen the cultural exchange and cooperation along the road of Maritime Silk Road. This is an important opportunity for the development of Chaozhou Opera. Chaozhou Opera is a bright pearl in Chinese traditional art and culture, also the outstanding representative of Chaoshan culture. In hundreds of years ago, Chaozhou Opera encouraged the Chaoshan ancestors to build their homes and bravely explore the world. Chaozhou Opera is one of the earliest operas to go abroad, and has produced tremendous influence, becoming the spiritual wealth and cultural symbol shared by more than 20 million Chaoshan people at home and abroad. As early as the mid-17th century, Chaozhou Opera came to Thailand, took root in this land, and blended with Thai culture, which promoted the exchange and development of culture and art between China and Thailand, and made Chaozhou Opera become the link and bridge of urging the kinship. Now Chaozhou Opera in Thailand needs to seize the opportunity of the times and recasts its glory. In front of the opportunity, only by continuing to improve the quality influence can Chaozhou Opera obtain more support to alleviate the trouble in the process of the spread of Chaozhou Opera in Thailand. Just as the Zhuang Mellon had mentioned in an interview that in the cause of the spread of Chaozhou Opera in Thailand, he always wanted to set up a opera school to train the younger generation but he didn't t have enough money. Now for many Chaozhou Operatroupesin Thailand, the economic source most relies on some Chaozhou Associations and other civil society 
charitable contributions, as well as a small amount of income from performing. Therefore, if Chaozhou Opera wants to get sustainable development in Thailand, it must seize the opportunity to improve its influence and seek more support.

\section{CONCLUSION}

To sum up, Chaozhou Opera, with a long history, has a unique status for Chinese people in Thailand. It is the sustenance of nostalgia, also the link of national culture. However, with the development of the times, Chaozhou Opera will inevitably encounter problems in the process of the spread. But the strategy of the 21st Century Maritime Silk Road gives Chaozhou Opera another opportunity to take off. At present, with the complex changes in the world, the strategy of the 21st Century Maritime Silk Road is a powerful means for China to create a cooperative, peaceful and harmonious environment in which China can get a good opportunity and external environment to fully deepen the reform. In this process, culture should bear the role of a link to promote the communication and trust along the road. The spread of Chaozhou Opera in Thailand must actively overcome difficulties, strive to innovate, and adhere to the principle of "Going out" and, "Coming in" to expand the influence and seek more support so that it can bear the mission of the times.

\section{Funding}

This project was supported by Youth innovation talent project of Guangdong University of Foreign Studies Center of Foreign Literature and Culture, China (Program number: 16QNCX10).

This project was supported by scientific research project of Guangdong University of Foreign Studies , China (Program number:17QN27).

\section{REFERENCES}

[1] Baker, Chris and Phongpaichit,Pasuk. (2005). A History of Thailand. Cambridge: Cambridge University Press.

[2] Banks, David J. (1976). Changing identities in Modern Southeast Asia. Paris: Mouon Publishers

[3] Chou, Cynthia and Houben, Vincent. (2006). Southeast Asian Studies:Debates and New Directions. Singapore:Institute of Southeast Asian Studies.

[4] Flood, Thadeus.(1978.).The Dynastic Chronicles Bangkok Era:the First Reign:volume one. Tokyo: The Center for East Asian Cultural Studies.

[5] Gorter, Cees, Ni jkamp, Peter and Poot, Jacque.(1998).Crossing Borders: Regional and Urban Perspectives on International Migration. London: Ashgate Publishing LTD.

Lei Miao was born in He Nan, China, in 1981. He is currently a lecturer in the School of interpreting \& translation Studies, Guangdong University of foreign studies, Guang Zhou, China. His research interests include Western Culture and Chinese literature.

Qiuping Wang was born in Si Chuan, China in 1985. She received her PH.D. degree in comparative culture from Guangdong University of foreign studies, China in 2017. She is currently a lecturer in the Faculty of Chinese Language and Culture, Guangdong University of foreign studies, Guang Zhou, China. Her research interests include Western Culture and Chinese literature. 